\title{
Heterogeneity in deformation of granular ceramics under dynamic loading
}

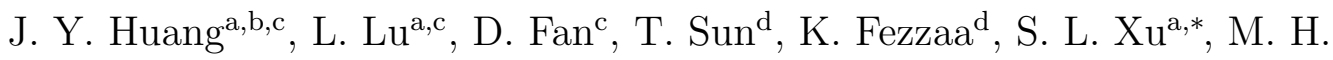 \\ Zhu ${ }^{\mathrm{b}, *}$, S. N. Luo ${ }^{\mathrm{c}, \mathrm{b}, *}$ \\ ${ }^{a}$ CAS Key Laboratory of Materials Behavior and Design, Department of Modern \\ Mechanics, University of Science and Technology of China, Hefei, Anhui 230027, P. R. \\ China \\ ${ }^{b}$ Key Laboratory of Advanced Technologies of Materials, Ministry of Education, \\ Southwest Jiaotong University, Chengdu, Sichuan 610031, P. R. China \\ ${ }^{c}$ The Peac Institute of Multiscale Sciences, Chengdu, Sichuan 61020\%, P. R. China \\ ${ }^{d}$ Advanced Photon Source, Argonne National Laboratory, Argonne, Illinois 60439, USA
}

\begin{abstract}
Dynamic compression experiments are conducted on micron-sized SiC powders of different initial densities with a split Hopkinson bar. Digital image correlation is applied to images from high-speed x-ray phase contrast imaging to map dynamic strain fields. The x-ray imaging and strain field mapping demonstrate the degree of heterogeneity in deformation depends on the initial powder density; mesoscale strain field evolution is consistent with softening or hardening manifested by bulk-scale loading curves. Statistical analysis of the strain probability distributions exhibits exponential decay tail similar to those of contact forces, which are supposed to lead to the grain-scale heterogeneity of granular materials.
\end{abstract}

Keywords: Powder processing; Dynamic compaction; X-ray imaging;

\footnotetext{
*Corresponding author

Email addresses: slxu99@ustc.edu.cn (S. L. Xu ), zhuminghao@swjtu.cn (M. H. Zhu ), sluo@pims.ac.cn (S. N. Luo)
} 
Digital image correlation; Heterogeneous deformation

Impact-induced compaction and/or sintering of powders is an important approach for synthesizing bulk ceramics, metals, alloys and composites with improved mechanical properties [1,2], and widely used in, for example, powder metallurgy $[1,3,4]$. The formation of a high quality compact depends on a number of factors, and identifying the important ones can help reduce undesired microstructures, including large density variations and internal cracks [5]. High strain-rate $\left(10^{2}-10^{3} \mathrm{~s}^{-1}\right)$ dynamic compression of granular materials, such as sand and soil, is also of interest in civil engineering $[6,7]$. Obtaining spatially and temporally resolved compaction dynamics in highly heterogeneous granular materials, is critical to understanding deformation mechanisms and developing constitutive models for powder compaction [5], but has been an experimental challenge.

For dynamic compression or high strain-rate loading in general, split Hopkinson pressure bar (SHPB) has been widely used for various materials including granular materials $[8,7]$. Strain gauges are effective for obtaining bulk, rather than meso-scale, responses. Local deformation dynamics can be characterized with two-dimensional (2D) strain field mapping, using optical digital image correlation [9] or x-ray digital image correlation (XDIC) $[10,11]$. XDIC is advantageous for the penetration capabilities of x-rays, and relies on images acquired with such techniques as x-ray phase contrast imaging (XPCI) $[12,13,14]$. XPCI is particularly useful to image low- $Z$ powders including $\mathrm{SiC}$, and the particles naturally serve as speckles. While density or particle displacement distributions under quasi-static loading have been examined to certain detail $[15,16]$, measurements on dynamic strain 
distributions in ceramic powders with high-speed XDIC are extremely rare. Shock compaction experiments on powders usually yield bulk-scale stressdensity relations $[17,18,4]$ and grain-scale deformation dynamics is largely untouched [10]. Heterogeneous force distribution in granular materials (force chains) has been widely observed $[19,20]$. Nonetheless, heterogeneity in deformation has not been fully investigated from a quantitative point of view.

In the present study, high strain-rate compression experiments are conducted on micron-sized $\mathrm{SiC}$ powders of different initial densities with SHPB and high-speed XDIC. The x-ray imaging and strain field mapping demonstrate that the degree of heterogeneity in deformation depends on the initial density of a powder, and mesoscale strain field evolution is consistent with softening or hardening manifested by the bulk-scale compression curves. Statistical analysis of the strain fields reveals that the strain probability distribution follows an exponential form, similar to contact force distribution within the context of force chains [19].

$\mathrm{SiC}$ powder with a mean particle size of $\sim 15 \mu \mathrm{m}$ (Fig. 1 inset) is chosen for our dynamic compaction experiments. As shown in Fig. 1, the SiC powder (7) is sandwiched between two thin polymethylmethacrylate or PMMA plates (6). The dimensions of the sample perpendicular to the x-ray (8) direction are $4 \mathrm{~mm} \times 5 \mathrm{~mm}$, and the thickness along the view direction is 0.5 mm. We implement a mini SHPB device along with a high-speed x-ray imaging system $(8,9,10)$ at the beamline 32-ID of the Advanced Photon Source. Details of the imaging system were presented previously [13]. The striker (1), incident bar (2) and transmission bar (3) of the SHPB are all made of high-strength steel with a diameter of $4 \mathrm{~mm}$. After the SHPB's gas gun is 


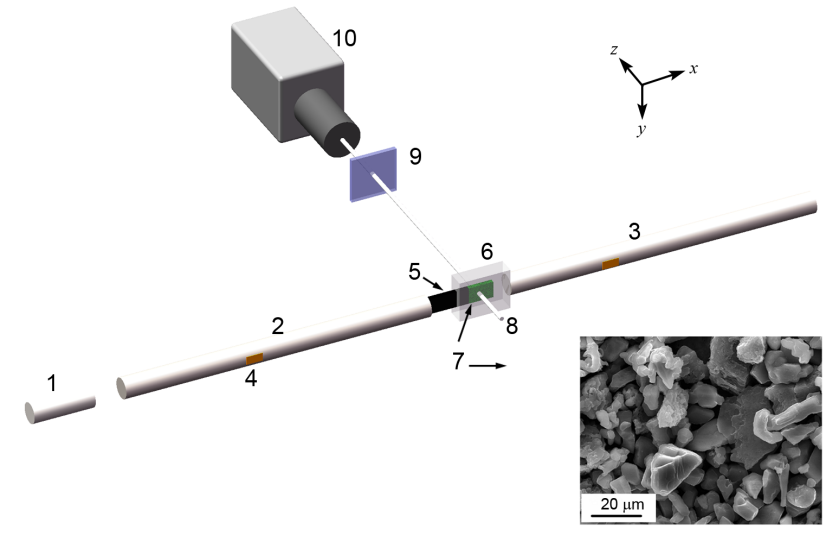

Figure 1: Schematic diagram of the SHPB loading and high-speed x-ray imaging system. 1: striker; 2: incident bar; 3: transmission bar; 4: strain gauges; 5: driver plate; 6: PMMA sample container; 7: powder sample; 8: x-ray beam; 9: scintillator; 10: high-speed camera. Inset: a micrograph of the $\mathrm{SiC}$ particles prior to impact loading.

fired, impact of the striker on the incident bar generates an elastic wave propagating through the incident bar (along the $x$ direction, Fig. 1). When the incident wave arrives at the interface between the incident bar and the steel driver plate (5), it is partially reflected owing to impedance mismatch, while the rest is transmitted into the transmission bar. The incident, reflected and transmitted waves are recorded by strain gages (4). The transmitted wave is used to calculate the stress applied to the sample $\left(\sigma_{\mathrm{s}}\right)[7]$, and

$$
\sigma_{\mathrm{s}}=E_{\mathrm{b}} \varepsilon_{\mathrm{t}} \frac{A_{\mathrm{b}}}{A_{\mathrm{s}}}
$$

where $\varepsilon$ is strain, $E$ is Young's modulus, $A$ is cross-section area, and subscripts $\mathrm{t}$ and $\mathrm{b}$ denote the transmission bar and sample, respectively.

Since uniform deformation is usually difficult to achieve in soft/porous materials under SHPB loading, the strain calculated with incident and transmitted waves is not accurate [7]. Therefore, only stress-time curves are pre- 
sented and discussed where appropriate. The incident wave signal is mainly used to trigger the high-speed camera (10). Upon loading, the x-rays transmitted through the powder sample form images on the scintillator (9) which are captured by the high-speed camera as image sequences.

Figure 2(a) shows the stress-time curves of the two samples with different initial densities, $\rho_{0}=1.86 \mathrm{~g} / \mathrm{cm}^{3}$ (densely packed, sample A) and $1.42 \mathrm{~g} / \mathrm{cm}^{3}$ (loosely packed, sample B). These two samples exhibit different compression responses. The axial stress in sample A increases approximately monotonically with time $(t)$. However, the stress evolution for sample B displays three distinct stages, similar to those of aluminum foams [21]. For $t \leq 15 \mu \mathrm{s}$, the curve for sample B coincides with that of sample A, showing a linear increase as expected for elastic deformation in a normal solid (stage I). The difference in wave speed due to that in the initial density is minor on the time scales of current experiments. Then the stress becomes approximately constant and even drops until a rebound at $25-30 \mu$ s (stage II), followed by a slow rise (stage III). However, the final stress level is significantly lower than that in the denser sample A.

High-speed image sequences are acquired during compression. The camera frame rate is set at $180,000 \mathrm{~s}^{-1}$ and the exposure time is $0.35 \mu \mathrm{s}$. The spatial resolution is about $2 \mu \mathrm{m}$. The central area $(\sim 0.5 \mathrm{~mm} \times 0.5 \mathrm{~mm})$ of the sample is chosen for imaging. The powder particles cannot be exactly distinguished from each other because there are about 30 layers of particles in the $\mathrm{x}$-ray direction (the $z$-axis). The loading direction is along the $x$-axis. Since the x-ray intensity is not uniform across the plane of observation (the $x y$-plane), a flat-field correction procedure is applied to all images for bet- 
(a)

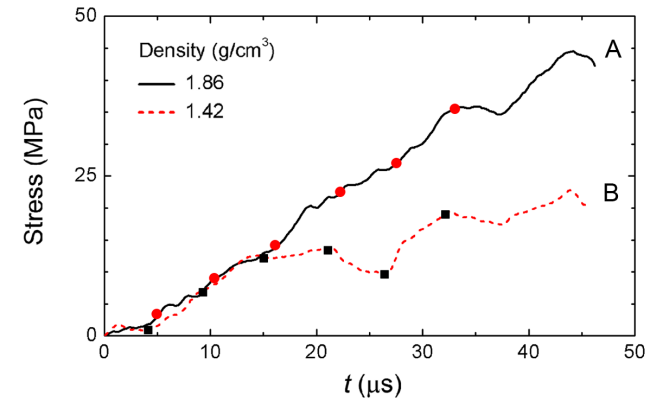

y

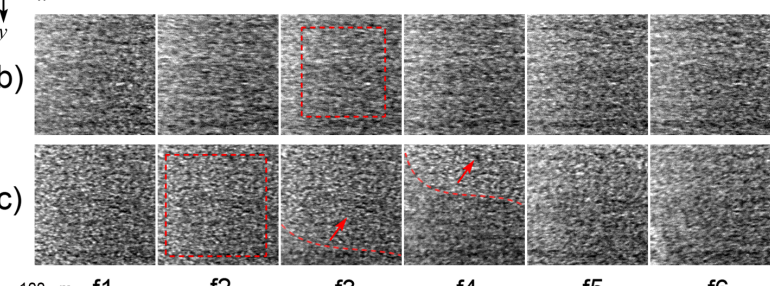

$\stackrel{100 \mu m}{L}$ f1

f2

f3

f4

f5

f6

Figure 2: Stress-time curves of samples with high (sample A) and low (sample B) initial densities (a), and snapshots for samples A (b) and B (c) during compression. Frames f1-f6 correspond to the instants (circles for sample A and squares for B) in (a). The frame intervals are $5.6 \mu \mathrm{s}$. The coordinate system is defined in Fig. 1. The arrows indicate the propagation of a compaction front, marked by the dashed curves. The dashed rectangles indicate the ROIs for XDIC analyses. 
ter DIC analyses [22]. The transmitted intensity field $I(x, y)$ is corrected to $I_{\mathrm{c}}(x, y)$ via

$$
I_{\mathrm{c}}(x, y)=\frac{I(x, y)}{I_{0}(x, y)} I_{0}^{*},
$$

where $I_{0}(x, y)$ is the incident x-ray intensity field acquired without a sample in place, and $I_{0}^{*}$ is the mean of the incident intensity field.

The snapshots at different instants (frames f1-f6, noted in Fig. 2(a)) after flat-field correction are shown in Figs. 2(b) and 2(c) for samples A and $\mathrm{B}$, respectively. The particles in sample $\mathrm{A}$ are compacted uniformly across the sample. However, we observe in sample B a compaction front, propagating from the lower left to the upper right (indicated by the dashed curves in frames $\mathrm{f} 3$ and $\mathrm{f4}$ ). Nonuniform compaction features appear to be more obvious in the movie (see Sumpllementary Materials), but are clearly identified in XDIC analyses as discussed below. The propagation velocity of the compaction front $\left(v_{\mathrm{cf}}\right)$ is estimated to be $\sim 40 \mathrm{~m} / \mathrm{s}$, much higher than the impact velocity $(\sim 5 \mathrm{~m} / \mathrm{s})$. The moment when the compaction band appears in the sample actually corresponds to the inflection point of the loading curve of sample B (f3). The compaction band sweeps across the sample during the subsequent period of $\sim 15 \mu \mathrm{s}$ (f3-f6), corresponding to stage II on the loading curve (Fig. 2(a)). Thus, the macro stress relaxation is attributed to localized deformation in sample B. The compaction band is similar to that observed in aluminum foams [23] and porous rocks [24], which formed mainly due to inherent pore collapse. However, the intrinsic mechanism in our case is quite different, given unique microstructures of granular materials. The compaction band is not strictly parallel to the loading direction, probably caused by heterogeneities in microstructures and stress transmission of the 
granular powders.

To better understand deformation dynamics in compressed SiC powders, XDIC [10] is used to map strain fields in the powder samples. The dashed rectangles are the selected regions of interest (ROIs) for XDIC analyses. The particles yield natural speckles on the images formed via transmitted xrays. Edge enhancement by XPCI facilitates DIC analyses without additional marker particles as utilized in literature [5, 15]. Correlation analyses are carried out between adjacent frames (e.g., f3 and f4 in Fig. 2(b)). The Lagrangian normal strains $\left(E_{x x}, E_{y y}\right)$ and shear strain $\left(E_{x y}\right)$ are calculated from the displacement $(u)$ gradients as

$$
E_{i j}=\frac{1}{2}\left(u_{i, j}+u_{j, i}+u_{k, i} u_{k, j}\right),
$$

where $i, j=x, y$. The von Mises equivalent strain $\left(E_{\mathrm{eq}}\right)$ is calculated from the above strain quantities as

$$
E_{\text {eq }}=\frac{\sqrt{2}}{3}\left[E_{x x}^{2}+E_{y y}^{2}+\left(E_{x x}-E_{y y}\right)^{2}+6 E_{x y}^{2}\right]^{\frac{1}{2}} .
$$

The strain fields $\left(E_{x x}, E_{y y}, E_{x y}\right.$ and $\left.E_{\text {eq }}\right)$ in sample A obtained from frames $\mathrm{f} 3$ and $\mathrm{f} 4$ are presented in Fig. 3(a). Heterogeneous 3D strain fields are induced in the powder samples, although overall, the sample undergoes bulk 1D-strain loading since it is constrained along the $y$ - and $z$-axes. The local and global maximum amplitudes of $E_{x x}(x, y)$ are much higher than the $E_{y y}$ counterparts. $E_{y y}(x, y)$ is largely induced by local Poisson's effects. Significant shear strain $E_{x y}$ indicates that complex particle rearrangement, including contact sliding and particle rotation, takes place in the particle assembly during dynamic loading. The apparent strain localization networks 


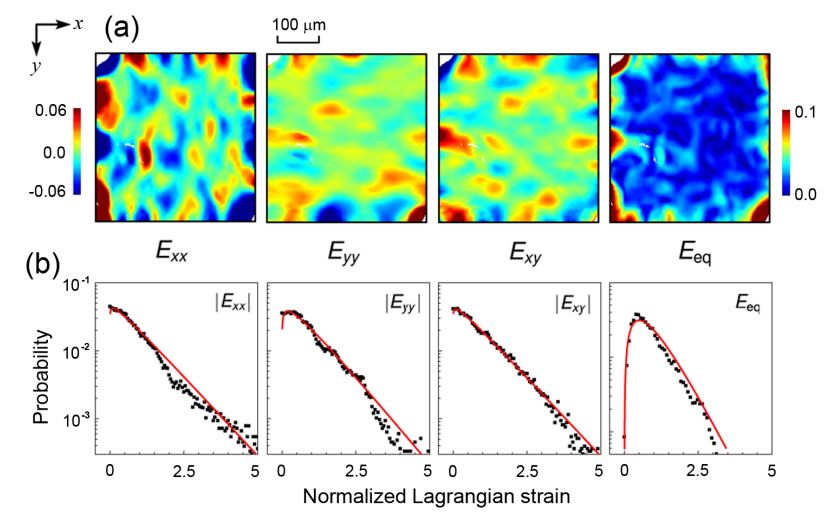

Figure 3: (a) 2D strain fields for sample A at frame f4, and (b) corresponding probability vs normalized Lagrangian strain curves. The coordinate system is defined in Fig. 1. The color bar for $E_{x x}, E_{y y}$ and $E_{x y}$ is presented on the left side of (a) while that for $E_{\text {eq }}$ is on the right.

may be attributed to the force chains in granular materials [25, 20].

To verify this hypothesis, we calculate the probability $(P)$ as a function of normalized strain amplitudes $\left(\overline{\left|E_{i j}\right|}\right)$, and the results are presented in Fig. 3(b). The probability distributions of the normalized Lagrangian strains, namely, $\left|E_{x x}\right| /\left\langle\left|E_{x x}\right|\right\rangle,\left|E_{y y}\right| /\left\langle\left|E_{y y}\right|\right\rangle,\left|E_{x y}\right| /\left\langle\left|E_{x y}\right|\right\rangle$, and $\left|E_{\text {eq }}\right| /\left\langle\left|E_{\text {eq }}\right|\right\rangle$, all exhibit similar features: a peak (for $\overline{\left|E_{\text {eq }}\right|}$ ) or plateau (for $\overline{\left|E_{x x}\right|}, \overline{\left|E_{y y}\right|}$ and $\overline{\left|E_{x y}\right|}$ ) at small strain values, followed by an exponential decay tail at larger strain values. Here $\langle\cdots\rangle$ denotes spatial average. These characteristics have been widely observed in the probability distributions of contact forces [20, 19], and the exponential tail can be explained by the $q$-model of force transmission [25]. The most common function form describing the probability distribution is $[19]$

$$
P\left(\overline{\left|E_{i j}\right|}\right)=a\left(\overline{\left|E_{i j}\right|}\right)^{\lambda} \exp \left\{-(1+\lambda) \overline{\left|E_{i j}\right|}\right\},
$$

where $\lambda$ characterizes the decay rate of probability at large strains, being 
0.10, 0.24, 0.09 and 1.19 for $\overline{\left|E_{x x}\right|}, \overline{\left|E_{y y}\right|}, \overline{\left|E_{x y}\right|}$ and $\overline{\left|E_{\text {eq }}\right|}$, respectively. It is interesting to note that $\lambda$ for $\overline{\left|E_{\text {eq }}\right|}$ is comparable to the typical value for contact forces, 0.90 [19]. The corresponding values of $a$, a normalization factor, are $0.06,0.07,0.06$ and 0.27 , respectively. The strain fields exhibit similar characteristics in the probability distributions to those in contact forces, leading support to the notion that heterogeneous deformation at grain scale is caused by heterogeneous contact force transmission in granular materials. The length scale of the force chains is several (3-5) particles wide [20], consistent with that of the heterogeneity in the strain maps $(\sim 60 \mu \mathrm{m}$ or 4 particles, Fig. 3(a)).

The strain maps $\left(E_{x x}, E_{y y}, E_{x y}\right)$ in sample B at three successive moments (frames $\mathrm{f} 2-\mathrm{f} 4$ ) are presented in Fig. 4. The correlation in white areas is too poor to render reliable strain calculations, as a result of large deformation which disfigures speckles. The strain fields in sample B at frame f2 are similar to those in sample A (Fig. 3(a)), showing both localized compression and tension, although the strain amplitudes are much smaller in sample B. The deformation is mainly accommodated by compression at particle contacts, while significant particle rearrangement does not occur. However, at frame f3, the strain fields are divided into two distinct regions (Fig. 4), which are also apparent in the raw image (Fig. 2(c)). Consistently, the compression curve shows a bimodal feature with considerable softening at f3 (15 $\mu$ s, Fig. 2(a)). Pronounced compression strain (ellipses) is induced at the lower left corner of the sample where the compaction band nucleates, as indicated by the dashed curve in Fig. 2(c), while the other region remains nearly undisturbed with minor strain. 
The boundary between these two regions is consistent with the moving front (dashed curves) identified in Fig. 2(c), which is thus confirmed to be a compaction front. This is the first time that the dynamic compaction band in granular materials is verified in experiments. Since the stress level is too low to break micron-sized ceramic particles, particle breakage is not expected in the sample [8]. The compaction band is produced mainly by pronounced particle rearrangement caused by the collapse of force chains when the loading stress exceeds the threshold. There also exist significant tensile and shear strains in the compaction band. It can be inferred that apart from particle translation, particle rotation also occurs in the compaction band, and plays an important role in deformation accommodation of granular materials [26]. At frame $\mathrm{f} 4$, the compaction front propagates forward and upward. The white regions increase markedly in area and strain amplitude with increasing loading stress, since the large deformation in the compaction band reduces image correlation considerably. Beyond frame $\mathrm{f} 4(t>30 \mu \mathrm{s})$, strain becomes more homogenized as the compaction front sweeps across the whole sample, the powder is densified, and the compression curve steepens up (Fig. 2(c)).

We have performed dynamic compression experiments on micron-sized $\mathrm{SiC}$ powders of different initial densities with a split Hopkinson bar, and high-speed x-ray imaging. Strain field mapping demonstrates that the degree of heterogeneity in deformation depends on the initial density of a powder. Heterogeneity is more pronounced in low density powders and demonstrated in the form of compaction bands. Marked localization in compression strains occurs with considerable compaction initiated via particle rearrangement, which consequently leads to softening (stress relaxation) or hardening 


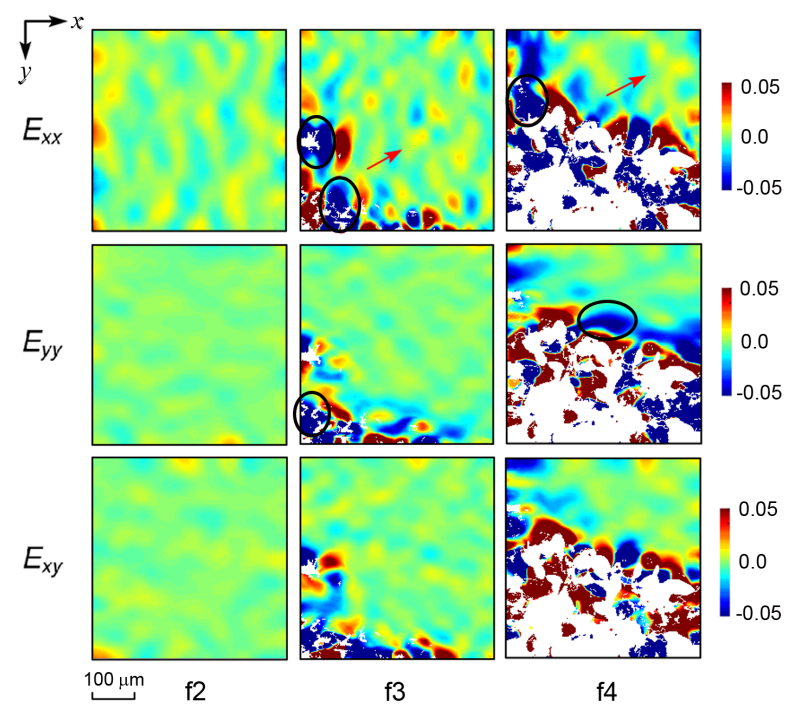

Figure 4: 2D strain fields for sample $\mathrm{B}$ at frames $\mathrm{f} 2-\mathrm{f} 4$. The arrows indicate the propagation of localized strain or compaction band, and the ellipses highlight the compression strain localizations. The coordinate system is defined in Fig. 1.

as manifested by the bulk-scale loading curves. Statistical analysis of the dynamic strain fields reveals that the strain probability distribution follows an exponential decay form, similar to contact force distributions previously observed. Given this connection, the heterogeneity in grain-scale deformation is attributed to heterogeneous force transmission, i.e., force chains, in granular materials.

This work is supported by the 973 Project and NSFC of China. Use of the Advanced Photon Source was supported by the U. S. Department of Energy, Office of Science, Office of Basic Energy Sciences, under Contract No. DE-AC02-06CH11357. 
[1] J. Wang, X. Qu, H. Yin, M. Yi, X. Yuan, Powder Technol. 192 (2009) 131-6.

[2] L. Huang, W. Z. Han, Q. An, W. A. Goddard, S. N. Luo, J. Appl. Phys. 111 (2012) $013508-$.

[3] Z. Yan, F. Chen, Y. Cai, Powder Technol. 208 (2011) 596-9.

[4] Z. Jin, K. Chen, J. Li, H. Zeng, S.-F. Cheng, J. Liu, Z. Wang, N. Thadhani, Acta Mater. 52 (2004) 2147-54.

[5] S. McDonald, L. Schneider, A. Cocks, P. Withers, Scr. Mater. 54 (2006) 191-6.

[6] B. Song, W. Chen, V. Luk, Mech. Mater. 41 (2009) 777-85.

[7] J. Huang, S. Xu, S. Hu, Mech. Mater. 68 (2014) 15-28.

[8] J. Huang, S. Xu, S. Hu, Int. J. Impact Eng. 59 (2013) 1-10.

[9] S. A. Hall, D. M. Wood, E. Ibraim, G. Viggiani, Granul. Matter 12 (2010) 1-14.

[10] L. Lu, D. Fan, B. X. Bie, X. X. Ran, M. L. Qi, N. Parab, J. Z. Sun, H. J. Liao, M. C. Hudspeth, B. Claus, K. Fezzaa, T. Sun, W. Chen, X. L. Gong, S. N. Luo, Rev. Sci. Instrum. 85 (2014) 076101.

[11] D. Fan, L. Lu, B. Li, M. L. Qi, J. C. E, F. Zhao, T. Sun, K. Fezzaa, W. Chen, S. N. Luo, Rev. Sci. Instr. 85 (2014) 113902.

[12] S. McDonald, N. Ravirala, P. Withers, A. Alderson, Scr. Mater. 60 (2009) 232-5. 
[13] M. Hudspeth, B. Claus, S. Dubelman, J. Black, A. Mondal, N. Parab, C. Funnell, F. Hai, M. Qi, K. Fezzaa, et al., Rev. Sci. Instru. 84 (2013) 025102.

[14] S. Cottrino, Y. Jorand, E. Maire, J. Adrien, Mater. Charact 81 (2013) 111-23.

[15] C. M. Kong, J. J. Lannutti, J. Am. Ceram. Soc. 83 (2000) 685-90.

[16] J. R. Valdes, F. L. Fernandes, I. Einav, Granul. Matter 14 (2012) 71-6.

[17] T. J. Vogler, M. Y. Lee, D. E. Grady, Int. J. Solids Struct. 44 (2007) $636 \mathrm{C} 658$.

[18] P. Counihan, A. Crawford, N. Thadhani, Mater. Sci. Eng. A 267 (1999) 26-35.

[19] J. Brujić, S. F. Edwards, I. Hopkinson, H. A. Makse, Phys. A 327 (2003) 201-12.

[20] T. S. Majmudar, R. P. Behringer, Nature 435 (2005) 1079-82.

[21] P. Wang, S. Xu, Z. Li, J. Yang, C. Zhang, H. Zheng, S. Hu, Mater. Sci. Eng. A 620 (2015) 253-61.

[22] V. Tiwari, M. A. Sutton, S. R. Mcneill, Exp. Mech. 47 (2007) 561-79.

[23] K. Issen, T. Casey, D. Dixon, M. Richards, J. Ingraham, Scr. Mater. 52 (2005) 911-5.

[24] K. Issen, J. Rudnicki, J. Geophys. Res. 105 (2000) 21529-36. 
[25] M. Da Silva, J. Rajchenbach, Nature 406 (2000) 708-10.

[26] J. Huang, S. Xu, S. Hu, Granul. Matter 17 (2015) 111-20. 